\title{
On characterizations of s-topological vector spaces
}

Shallu Sharma, Madhu Ram, Sahil Billawria and Tsering Landol

Department of Mathematics, University of Jammu JK-180006, India

Received: 20 August 2018, Accepted: 29 October 2018

Published online: 17 March 2019.

\begin{abstract}
In the paper [4], M. Khan et. al. introduced the s-topological vector spaces and studied several of their properties. In this paper, we continue their work and set forth some new properties of s-topological vector spaces.
\end{abstract}

Keywords: Semi-open sets, semi-closed sets, s-topological vector spaces.

\section{Introduction}

The notion of topological vector spaces is one of the most important tool in mathematics and due to nice properties, it earns a great importance in various branches of mathematics like fixed point theory, operator theory, variational inequalities, etc. In 2015, M. Khan et. al. [4] introduced the concept of s-topological vector spaces which is basically a generalization of topological vector spaces [6]. The main purpose of the present paper is to give some new properties and characterizations of s-topological vector spaces.

In 1963, N. Levine [7] introduced the notion of semi-open sets in topological spaces. He defines a set $S$ in a topological space $X$ to be semi-open if there exists an open set $U$ in $X$ such that $U \subseteq S \subseteq C l(U)$; or equivalently, a subset $S$ of $X$ is semi-open if $S \subseteq C l(\operatorname{Int}(S))$, where $\operatorname{Int}(S)$ and $C l(S)$ denote the interior of $S$ and the closure of $S$ respectively. In [1], the authors define a set $S$ in a topological space $X$ is semi-closed if and only if its complement is semi-open; or equivalently, $S$ is semi-closed in $X$ if $\operatorname{Int}(C l(S)) \subseteq S$. The semi-closure of a subset $S$ of $X$, denoted by $s C l(S)$, is the intersection of all semi-closed subsets of $X$ containing $S$. In other words, the semi-closure of a subset $S$ of $X$ is the smallest semi-closed subset of $X$ containing $S$. It is known that a set $S$ in $X$ is semi-closed if and only if $s C l(S)=S$. In [1], it is proved that $x \in s C l(S)$ if and only if $S \cap U \neq \emptyset$ for any semi-open set $U$ in $X$ containing $x$. A point $x \in X$ is called a semi-interior point of $S$ if there exists a semi-open set $U$ in $X$ such that $x \in U \subseteq S$. The set of all semi-interior points of $S$, denoted by $\operatorname{sint}(S)$, is called semi-interior of $A$. Equivalently, $\operatorname{sint}(A)$ is the largest semi-open subset of $A$ in $X$. The family of all semi-open (resp. semi-closed) sets in $X$ is denoted by $S O(X)$ (resp. $S C(X)$ ). We represent the set of real numbers by $\mathbb{R}$ and the set of complex numbers by $\mathbb{C}$. The notations $\varepsilon$ and $\delta$ denote negligibly small positive real numbers.

Also, we recall some more definitions that will be used in the sequel.

Definition 1. A subset $A$ of a topological space $X$ is called a semi-neighborhood of a point $x \in X$ if there exists a semi-open set $U$ in $X$ such that $x \in U \subseteq A$

Definition 2. A collection $\left\{U_{\alpha}: \alpha \in \Lambda\right\}$ of semi-open sets in a topological space $X$ is called a semi-open cover of a subset $B$ of $X$ if $B \subseteq \cup\left\{U_{\alpha}: \alpha \in \Lambda\right\}$ holds.

Definition 3. A topological space $X$ is called semi-compact [2] if every semi-open cover of $X$ has a finite subcover. A subset $B$ of a topological space $X$ is said to be semi-compact relative to $X$ if, for every collection $\left\{U_{\alpha}: \alpha \in \Lambda\right\}$ of semi- 
open subsets of $X$ such that $B \subseteq \cup\left\{U_{\alpha}: \alpha \in \Lambda\right\}$, there exists a finite subset $\Lambda_{0}$ of $\Lambda$ such that $B \subseteq \cup\left\{U_{\alpha}: \alpha \in \Lambda_{0}\right\}$. A subset $B$ of a topological space $X$ is said to be semi-compact if $B$ is semi-compact as a subspace of $X$.

Definition 4. A mapping $f: X \rightarrow Y$ from a topological space $X$ to a topological space $Y$ is called semi-continuous [7] if $f^{-1}(V)$ is semi-open in $X$, for each open set $V$ in $Y$. In other words, $f$ is semi-continuous if for each $x \in X$ and for each open neighborhood $V$ of $f(x)$ in $Y$, there exists a semi-open neighborhood $U$ of $x$ in $X$ such that $f(U) \subseteq V$.

Definition 5. [4] Let $L$ be a vector space over the topological field $K$, where $K=\mathbb{R}$ or $\mathbb{C}$ with standard topology. Let $\tau$ be a topology on $L$ such that the following two conditions are satisfied:

(1) For each $x, y \in L$ and each open neighborhood $W$ of $x+y$ in $L$, there exist semi-open neighborhoods $U$ and $V$ of $x$ and $y$ respectively in $L$ such that $U+V \subseteq W$ and

(2) For each $x \in L, \lambda \in K$ and each open neighborhood $W$ of $\lambda x$ in $L$, there exist semi-open neighborhoods $U$ of $\lambda$ in $K$ and $V$ of $x$ in $L$ such that $U . V \subseteq W$. Then the pair $\left(L_{(K)}, \tau\right)$ is called an s-topological vector space.

Now we present an example of s-topological vector spaces. Actually, this is an improvement of example 3.2 in the paper [4].

Example 1. Let $L=\mathbb{R}$ be the vector space of real numbers over the field $K$, where $K=\mathbb{R}$ with standard topology and the topology $\tau$ on $L$ be generated by the base $\mathscr{B}=\{(a, b),[c, d): a, b, c$ and $d$ are real numbers with $0<c<d\}$. We show that $\left(L_{(K)}, \tau\right)$ is an s-topological vector space. For which we have to verify the following two conditions:

(1) Let $x, y \in L$. Then, for open neighborhood $W=[x+y, x+y+\varepsilon)($ resp. $(x+y-\varepsilon, x+y+\varepsilon))$ of $x+y$ in $L$, we can opt for semi-open neighborhoods $U=[x, x+\delta)(\operatorname{resp} .(x-\delta, x+\delta))$ and $V=[y, y+\delta)(\operatorname{resp} .(y-\delta, y+\delta))$ of $x$ and $y$ respectively in $L$ such that $U+V \subseteq W$ for each $\delta<\frac{\varepsilon}{2}$.

(2) Let $x \in L$ and $\lambda \in K$. We have following cases:

Case (1). If $\lambda>0$ and $x>0$, then clearly $\lambda x>0$. Consider an open neighborhood $W=[\lambda x, \lambda x+\varepsilon)$ (resp. $(\lambda x-$ $\varepsilon, \lambda x+\varepsilon)$ ) of $\lambda x$ in $L$. We can choose semi-open neighborhoods $U=[\lambda, \lambda+\delta)(\operatorname{resp} .(\lambda-\delta, \lambda+\delta))$ of $\lambda$ in $K$ and $V=[x, x+\delta)($ resp. $(x-\delta, x+\delta))$ of $x$ in $L$ such that $U . V \subseteq W$ for each $\delta<\frac{\varepsilon}{\lambda+x+1}$.

Case (II). If $\lambda<0$ and $x<0$, then $\lambda x>0$. So, for open neighborhood $W=[\lambda x, \lambda x+\varepsilon)(\operatorname{resp}$. $(\lambda x-\varepsilon, \lambda x+\varepsilon))$ of $\lambda x$ in $L$, we can choose semi-open neighborhoods $U=(\lambda-\delta, \lambda](\operatorname{resp} .(\lambda-\delta, \lambda+\delta))$ of $\lambda$ in $K$ and $V=(x-\delta, x]$ (resp. $(x-\delta, x+\delta)$ ) of $x$ in $L$ such that $U . V \subseteq W$ for sufficiently appropriate $\delta \leq \frac{-\varepsilon}{\lambda+x-1}$.

Case (III). If $\lambda=0$ and $x>0$ ( resp. $\lambda>0$ and $x=0$ ). Then $\lambda x=0$. Therefore, for any open neighborhood $W=(-\varepsilon, \varepsilon)$ of 0 in $L$, we can opt for semi-open neighborhoods $U=(-\delta, \delta)($ resp. $U=(\lambda-\delta, \lambda+\delta)$ ) of $\lambda$ in $\mathbb{R}$ and $V=(x-\delta, x+\delta)$ (resp. $V=(-\delta, \delta)$ ) of $x$ in $L$ such that $U . V \subseteq W$ for each $\delta<\frac{\varepsilon}{x+1}$ (resp. $\delta<\frac{\varepsilon}{\lambda+1}$ ).

Case (IV). If $\lambda=0$ and $x<0$ (resp. $\lambda<0$ and $x=0$ ). Then, for the selection of semi-open neighborhoods $U=$ $(-\delta, \delta)$ (resp. $U=(\lambda-\delta, \lambda+\delta)$ ) of $\lambda$ in $\mathbb{R}$ and $V=(x-\delta, x+\delta)$ (resp. $V=(-\delta, \delta)$ ) of $x$ in $L$, we have $U . V \subseteq W=(-\varepsilon, \varepsilon)$ for every $\delta<\frac{\varepsilon}{1-x}$ (resp. $\left(\delta<\frac{\varepsilon}{1-\lambda}\right)$ ).

Case (V). If $\lambda=0$ and $x=0$. Consider any open neighborhood $W=(-\varepsilon, \varepsilon)$ of 0 in $L$, we can find semi-open neighborhoods $U=(-\delta, \delta)$ of $\lambda$ in $\mathbb{R}$ and $V=(-\delta, \delta)$ of $x$ in $L$, we have $U . V \subseteq W$ for each $\delta<\sqrt{\varepsilon}$.

Thus, $\left(L_{(\mathbb{R})}, \tau\right)$ is an s-topological vector space.

\section{Characterizations}

Henceforth, an s-topological vector space $L$ means an s-topological vector space $\left(L_{(K)}, \tau\right)$ and by a scalar, we mean an element of $K$. Now we obtain some useful characterizations of s-topological vector spaces.

Theorem 1. Let A be any subset of an s-topological vector space L. The following assertions are valid:

(a) $x+\operatorname{sCl}(A) \subseteq C l(x+A)$ for each $x \in L$.

(b) $\operatorname{sCl}(x+A) \subseteq x+C l(A)$ for each $x \in L$. 
(c) $x+\operatorname{Int}(A) \subseteq \operatorname{sint}(x+A)$ for each $x \in L$.

(d) $\operatorname{Int}(x+A) \subseteq x+\operatorname{sint}(A)$ for each $x \in L$.

Proof. (a) Let $z \in x+s C l(A)$. Then $z=x+y$ for some $y \in s C l(A)$. Let $W$ be any open neighborhood of $z$. Then, by definition of s-topological vector spaces, there exist semi-open neighborhoods $U$ of $x$ and $V$ of $y$ in $L$ such that $U+V \subseteq W$. Since $y \in s C l(A)$, there is $g \in A \cap V$. Now $x+g \in(x+A) \cap(U+V) \subseteq(x+A) \cap W \Rightarrow(x+A) \cap W \neq \emptyset$. Therefore, $z \in C l(x+A)$. This proves that $x+s C l(A) \subseteq C l(x+A)$.

(b) Assume $y \in \operatorname{sCl}(x+A)$. Let $z=(-x)+y$ and let $W$ be any open neighborhood of $z$ in $L$. Since $L$ is an s-topological vector space, there exist semi-open neighborhoods $U$ and $V$ of $-x$ and $y$ respectively, in $L$ such that $U+V \subseteq W$. Since $y \in s C l(x+A), V \cap(x+A) \neq \emptyset$. So, there is $g \in V \cap(x+A)$. Now $-x+g \in(-x+x+A) \cap(U+V)=A \cap(U+V) \subseteq$ $A \cap W$ showing that $A \cap W \neq \emptyset$. Hence $-x+y \in C l(A)$; that is $y \in x+C l(A)$. Therefore, $s C l(x+A) \subseteq x+C l(A)$.

(c) Suppose that $y \in x+\operatorname{Int}(A)$. Then $-x+y \in \operatorname{Int}(A)$. Therefore, there exist semi-open sets $U$ in $L$ containing $-x$ and $V$ in $L$ containing $y$ such that $U+V \subseteq \operatorname{Int}(A)$. In particular, $-x+V \subseteq \operatorname{Int}(A) \subseteq A$ and, as a consequence, $V \subseteq x+A$. Therefore, $y \in \operatorname{sint}(x+A)$. Hence $x+\operatorname{Int}(A) \subseteq \operatorname{sint}(x+A)$.

(d) Suppose that $z \in \operatorname{Int}(x+A)$. Then $z=x+y$ for some $y \in A$. By definition of s-topological vector spaces, there exist $U, V \in S O(L)$ such that $x \in U, y \in V$ and $U+V \subseteq \operatorname{Int}(x+A)$. Consequently, $z=x+y \in x+V \subseteq x+\operatorname{sint}(A)$. Therefore, $\operatorname{Int}(x+A) \subseteq x+\operatorname{sint}(A)$.

Remark. The reverse inclusions do not hold in any part of the theorem above. We account for part (a) of this theorem. Counterexamples for other parts follow analogously. Consider example 1 , take $A=(0,1)$ and $x=1$ in $L$. Then $C l(x+A)=$ $[1,2)$ but $x+s C l(A)=(1,2)$. Hence the inclusion $C l(x+A) \subseteq x+s C l(A)$ fails to hold in $L$.

If we notice carefully, we observe that Theorem 1 is completely based on the first condition of the definition of s-topological vector spaces. The analog of this theorem which is based on the second condition of the same definition is the following result:

Theorem 2. Let A be any subset of an s-topological vector space L. Then the following statements hold:

(a) $\lambda s C l(A) \subseteq C l(\lambda A)$ for every non-zero scalar $\lambda$.

(b) $\operatorname{sCl}(\lambda A) \subseteq \lambda C l(A)$ for every non-zero scalar $\lambda$.

(c) $\operatorname{Int}(\lambda A) \subseteq \lambda \operatorname{sInt}(A)$ for every non-zero scalar $\lambda$.

(d) $\lambda \operatorname{Int}(A) \subseteq \operatorname{sint}(\lambda A)$ for every non-zero scalar $\lambda$.

Theorem 3. Let $A$ be any open subset of an s-topological vector space $L$. Then $x+A \subseteq C l(\operatorname{Int}(x+A))$ for each $x \in L$.

Proof. Straightforward.

Theorem 4. Let $F$ be any closed subset of an s-topological vector space L. Then Int $(C l(x+F)) \subseteq x+F$ for each $x \in L$.

Proof. Straightforward.

Definition 6. A topological space $X$ is said to be P-regular [5] if for each semi-closed set $F$ and $x \notin F$, there exist disjoint open sets $U$ and $V$ such that $x \in U$ and $F \subseteq V$.

Theorem 5. Let L be a P-regular s-topological vector space. Then the algebraic sum of any semi-compact set and closed set in L is closed.

Proof. let $A$ be a semi-compact and $F$ be a closed subset of $L$. We have to show that $A+F$ is closed set in $L$. For, let $x \notin A+F$. Then $\forall a \in A, x \notin a+F$. Since $F$ is closed in $L$ and translation of a closed set in s-topological vector spaces is semi-closed, we find that $a+F$ is semi-closed in $L$. As $L$ is P-regular, there exist open sets $U_{a}$ and $V_{a}$ such that $x \in U_{a}$, $a+F \subseteq V_{a}$ and $U_{a} \cap V_{a}=\emptyset$. Therein we find that $a \in V_{a}-F$ and hence $A \subseteq \cup_{a \in A}\left(V_{a}-F\right)$. Since any union of semi-open sets is semi-open, by theorem 3.4 of [4], $V_{a}-F=\cup_{b \in F}\left(V_{a}-b\right)$ is semi-open set in $L$. Consequently, by semi-compactness of $A$, there exists a finite subset $S$ of $A$ such that $A \subseteq \cup_{x \in S}\left(V_{x}-F\right)$. Let $U=\cap_{x \in S} U_{x}$, then $U$ is an open 
neighborhood of $x$ such that $U \cap(A+F)=\emptyset$.

If $y \in U \cap(A+F)$, then $y \in U_{x} \cap V_{x}$ for some $x \in S$, which is a contradiction to the fact that $U_{a} \cap V_{a}=\emptyset$, for each $a \in A$. thereby we find that $x \notin C l(A+F)$. This gives $C l(A+F)=A+F$. Hence $A+F$ is closed in $L$.

Next, we investigate further properties of s-topological vector spaces by using their basic idea.

Theorem 6. Let $A$ be any subset of an s-topological vector space $L$. Then $s C l(x+s C l(A)) \subseteq x+C l(A)$ for each $x \in L$.

Proof. Let $y \in \operatorname{sCl}(x+s C l(A))$. Consider $z=-x+y$ and let $W$ be an open neighborhood of $-x+y$ in $L$. Then there exist semi-open sets $U$ and $V$ in $L$ containing $-x$ and $y$ respectively, such that $U+V \subseteq W$. Since $y \in s C l(x+s C l(A))$, $V \cap(x+s C l(A)) \neq \emptyset$. So, there is $g \in V \cap(x+s C l(A))$. Now $-x+g \in(-x+x+s C l(A)) \cap(U+V) \subseteq s C l(A) \cap W$ implies $s C l(A) \cap W \neq \emptyset$. Since $W$ is semi-open, $A \cap W \neq \emptyset$. Consequently, $-x+y \in C l(A)$, i.e. $y \in x+C l(A)$. Hence $s C l(x+s C l(A)) \subseteq x+C l(A)$.

Theorem 7. Let $A$ be any subset of an s-topological vector space $L$. Then $x+\operatorname{Int}(A) \subseteq \operatorname{sInt}(x+\operatorname{sint}(A))$ for each $x \in L$.

Proof. Let $y \in x+\operatorname{Int}(A)$. Then $-x+y \in \operatorname{Int}(A)$ and consequently, there exist semi-open neighborhoods $U$ and $V$ of $-x$ and $y$ respectively, in $L$ such that $U+V \subseteq \operatorname{Int}(A)$. Now $-x+V \subseteq U+V \subseteq \operatorname{Int}(A) \subseteq \operatorname{sInt}(A)$ implies that $V \subseteq x+\operatorname{sInt}(A)$. Since $V$ is semi-open in $L$, we have that $y \in V \subseteq \operatorname{sInt}(x+\operatorname{sint}(A))$. Therefore, $x+\operatorname{Int}(A) \subseteq \operatorname{sint}(x+\operatorname{sint}(A))$.

Comparing Theorem 6 with part (b) of Theorem 1 and Theorem 7 with part (c) of Theorem 1, we find that the former is a generalization and the later is an improvement of corresponding parts.

Theorem 8. Let $C$ be any semi-compact subset of an s-topological vector space $L$. Then $x+C$ is compact, for each $x \in L$.

Proof. Let $\left\{U_{\alpha}: \alpha \in \Lambda\right\}$ be an open cover of $x+C$. Then $x+C \subseteq \cup\left\{U_{\alpha}: \alpha \in \Lambda\right\} \Rightarrow C \subseteq \cup\left\{-x+U_{\alpha}: \alpha \in \Lambda\right\}$. By [4, Theorem 3.4], $-x+U_{\alpha}$ is semi-open in $L$, for each $\alpha \in \Lambda$. Consequently, by semi-compactness of $C$, there exists a finite subset $\Lambda_{0}$ of $\Lambda$ such that $C \subseteq \cup\left\{-x+U_{\alpha}: \alpha \in \Lambda_{0}\right\}$. Thereby we find that $x+C \subseteq \cup\left\{U_{\alpha}: \alpha \in \Lambda_{0}\right\}$. Hence the assertion follows.

Theorem 9. Let L be an s-topological vector space. Then scalar multiple of a semi-compact set is compact.

Proof. Let $C$ be a semi-compact set in $L$ and $\lambda \neq 0$ be any scalar. We have to show that $\lambda C$ is compact. For, let $\left\{U_{\alpha}: \alpha \in \Lambda\right.$, where $\Lambda$ is an indexed set $\}$ be an open cover of $\lambda C$. Then $\lambda C \subseteq \cup\left\{U_{\alpha}: \alpha \in \Lambda\right\}$. Thereby $C \subseteq \frac{1}{\lambda} \cdot \cup\left\{U_{\alpha}: \alpha \in \Lambda\right\}$. This implies that $C \subseteq \cup\left\{\frac{1}{\lambda} U_{\alpha}: \alpha \in \Lambda\right\}$. By [4, Theorem 3.4], $\frac{1}{\lambda} U_{\alpha}$ is semi-open. Consequently, $\left\{\frac{1}{\lambda} U_{\alpha}: \alpha \in \Lambda\right\}$ is a semi-open cover of $C$. But $C$ is semi-compact. Therefore, there exists a finite subset $\Lambda_{0}$ of $\Lambda$ such that $C \subseteq \cup\left\{\frac{1}{\lambda} U_{\alpha}: \alpha \in \Lambda_{0}\right\}$. This gives $\lambda C \subseteq \cup\left\{U_{\alpha}: \alpha \in \Lambda_{0}\right\}$ showing that $\lambda C$ is compact. This completes the proof.

Remark. Let $L$ and $T$ be s-topological vector spaces over the field $K$. A mapping $f: L \rightarrow T$ is said to be linear if $f(\alpha x+$ $\beta y)=\alpha f(x)+\beta f(y)$, for all $x, y \in L$ and $\alpha, \beta \in K$.

A mapping $f: L \rightarrow K$ is called linear functional if $f(\alpha x+\beta y)=\alpha f(x)+\beta f(y)$, for all $x, y \in L$ and $\alpha, \beta \in K$. The kernel of $f$ is defined by $\operatorname{Ker}(f)=\{x \in L: f(x)=0\}$.

Theorem 10. Let $f: L \rightarrow M$ be a linear map, where $L$ is an s-topological vector space and $M$ is a topological vector space. If $f$ is continuous at origin, then $f$ is semi-continuous everywhere.

Proof. Suppose that $f$ is continuous at origin. Let $0 \neq x \in L$ and $V$ be an open neighborhood of $f(x)$ in $M$. Then $V-f(x)$ is an open neighborhood of zero in $M$ because $M$ is a topological vector space. By continuity of $f$ at origin, there exists an open neighborhood $U$ of zero in $L$ such that $f(U) \subseteq V-f(x)$. By linearity of $f, f(x+U) \subseteq V$. Since $L$ is an s-topological vector space, $x+U$ is semi-open neighborhood of $x$ in $L$. Consequently, $f$ is semi-continuous at $x$ in $L$. Since $x$ is an arbitrary non-zero element of $L$, it follows that $f$ is semi-continuous at every non-zero element of $L$. Further, continuity of $f$ at origin implies that $f$ is semi-continuous at zero. Hence $f$ is semi-continuous everywhere. 
Corollary 1. Let $L$ be an s-topological vector space. Assume $0 \neq f: L \rightarrow K$ be a linear functional. If $f$ is continuous at origin, then $f$ is semi-continuous.

Theorem 11. Let $f: L \rightarrow K$ be a linear functional, where $L$ is an s-topological vector space. If $f$ is continuous at origin, then $\operatorname{Ker}(f)$ is semi-closed in $L$.

Proof. Suppose that $f$ is continuous at origin. If $f=0$, we are done. Assume $f \neq 0$. By virtue of corollary $1, f$ is semicontinuous. Since $K(\mathbb{R}$ or $\mathbb{C}$ ) is endowed with standard topology which is Hausdorff. Therefore, $\{0\}$ is closed in $K$. By semi-continuity of $f, f^{-1}(\{0\})$ is semi-closed in $L$. But $f^{-1}(\{0\})=\{x \in L: f(x)=0\}=\operatorname{Ker}(f)$. Hence the assertion follows.

\section{Acknowledgements}

The second author is supported by UGC-India under the scheme of NET-JRF Fellowship.

\section{Competing interests}

The authors declare that they have no competing interests.

\section{Author's contributions}

All authors have contributed to all parts of the article. All authors read and approved the final manuscript.

\section{References}

[1] S. G. Crossley and S. K. Hildebrand, Semi-closure, Texas J. Sci. 22(1971), 99-112.

[2] C. Dorsett, Semi-compactness, semi separation axioms, and product spaces, Bull. Malays. Math. Sci. Soc., 4(1) (1981), 21-28.

[3] J. L. Kelly, General Topology, Van Nastrand (New York 1955).

[4] M. D. Khan, S. Azam and M.S. Bosan, s-topological vector spaces, J. Linear. Topological. Algebra. 04(02) (2015), $153-158$.

[5] M. Khan and B. Ahmad, On p-regular spaces, Math. Today, 13 (1995), 51-56.

[6] Kolmogroff, Zur Normierbarkeit eines topologischen linearen Raumes, Studia Mathematica, 5(1934), 29-33.

[7] N. Levine, Semi-open sets and semi-continuity in topological spaces, Amer. Math. Monthly. 70(1963), 36-41.

[8] L. Narici and E. Beckenstein, Topological Vector Spaces, CRC Press 2010.

[9] T. Noiri, A note on s-normal spaces, Bull. Math. Soc. Sci. Math. R. S. Roumanie (N.S) 25 (73) (1981), 55-59.

[10] T. M. Nour, A Note on some Applications of Semi-open sets, Internat. J. Math. and Math. Sci. 21(1) (1998), 205-207. 